DOI: $10.15593 / 2499-9873 / 2020.4 .05$

УДК 004.89; 67.02

\title{
И.П. Селетков
}

Пермский государственный национальный исследовательский университет, Пермь, Россия

ПРИМЕНЕНИЕ МАТРИЧНОГО АППАРАТА НЕЧЕТКОЙ ЛОГИКИ ДЛЯ ПОДДЕРЖКИ ПРИНЯТИЯ РЕШЕНИЙ В ПРОЦЕССЕ ОБСЛУЖИВАНИЯ ТЕХНОЛОГИЧЕСКОГО ОБОРУДОВАНИЯ НЕФТЕДОБЫЧИ

Решается задача построения интеллектуальной системы поддержки принятия решений при обслуживании технологического оборудования нефтедобычи. На первом этапе - выбора интеллектуальной модели - показано, что в существующих условиях получение обучающей выборки в цифровом виде затруднительно. С другой стороны, есть возможность получить знания экспертов предметной области - мастеров и технологов - в виде набора лингвистических правил, на основании чего делается вывод об эффективности применения нечеткой логики для решения данной задачи.

На этапе построения интеллектуальной модели предлагается применение матричного аппарата нечеткой логики, производится его развитие алгоритмом нечеткого логического вывода на основании векторных нечетких предикатов, демонстрируются его возможности и преимущества. В частности, показывается, что матричное представление позволяет свести вычисления к решению системы линейных уравнений, в явном виде определить область значений анализируемых параметров, при которых база знаний не позволяет сделать нечеткие заключения.

Для анализа ретроспективной информации по изменению значений параметров технологического оборудования с течением времени предлагается модель нечеткого логического автомата в виде нечеткой комбинационной схемы, анализирующей внешний блок памяти. Показывается, в каких случаях можно осуществить переход от автомата к комбинационной схеме, каким образом это можно сделать. Основным преимуществом этого подхода является отсутствие необходимости использования трудноформализуемого понятия нечеткого состояния, что ведет к упрощению построения нечетких логических устройств с памятью.

В завершение работы делаются краткие выводы о применении предлагаемых методов и алгоритмов для построения системы поддержки принятия решений, ее тестировании, внедрении и эффрективности.

Ключевые слова: нечеткая логика, матричный аппарат нечеткой логики, нечеткие логические операции, нечеткие предикаты, нечеткий логический вывод, нечеткие базы знаний, нечеткая комбинационная логика, нечеткие логические автоматы, система поддержки принятия решений, оборудование нефтедобычи. 


\author{
I.P. Seletkov \\ Perm State University, Perm, Russian Federation \\ APPLICATION OF MATRIX APPROACH \\ OF FUZZY LOGIC FOR DECISION SUPPORT \\ IN OIL MINING EQUIPMENT SERVICE
}

\begin{abstract}
The work solves the problem of building an intelligent decision support system for servicing oil production equipment. At the first stage - the choice of an intelligent model - it is shown that in the existing conditions it is difficult to obtain a training sample in digital form. On the other hand, there is an opportunity to gain knowledge of subject matter experts - masters and technologists - in the form of a set of linguistic rules. Based on this, a conclusion about the effectiveness of the use of fuzzy logic to solve this problem is made.

At the stage of constructing an intelligent model, the use of the matrix approach of fuzzy logic is proposed. To elaborate this approach an algorithm of fuzzy inference based on vector fuzzy predicates is developed. Capabilities and advantages of new algorithm are demonstrated. In particular, it is shown that the matrix representation makes possible reducing computations to solving a system of linear equations. Matrix inference also allows to explicitly determine the range of values of the analyzed parameters at which the knowledge base does not allow making a clear conclusion.

A model of a fuzzy logic machine in the form of a fuzzy combinational circuit that analyzes an external memory block is proposed for the analysis of retrospective information on the change in the values of the parameters of technological equipment over time. Specific cases allowing the transition from a state machine to a combinational circuit are shown. Article also shows how this can be done. The main advantage of this approach is the absence of the need to use the difficult to formalize concept of a fuzzy state, which leads to a simplified construction of fuzzy logical devices with memory.

At the end work contains brief conclusions about the application of the proposed methods and algorithms for building, testing, implementing a decision support system and about its effectiveness.

Keywords: fuzzy logic, matrix approach of fuzzy logic, fuzzy logic operators, fuzzy predicates, fuzzy inference, fuzzy knowledge base, fuzzy combination logic, fuzzy logical automata, decision support system, oil mining equipment.
\end{abstract}

\title{
Введение
}

В настоящее время в соответствии с Указом Президента РФ ${ }^{1}$ одним из приоритетных и перспективных направлений научнотехнологического развития РФ является «переход к передовым цифровым, интеллектуальным производственным технологиям, роботизированным системам, новым материалам и способам конструирования, создание систем обработки больших объемов данных, машинного обучения и искусственного интеллекта».

С целью реализации данной концепции во многих отраслях промышленности внедряются современные системы автоматизированного управления производством и системы поддержки принятия решений

${ }^{1}$ О стратегии научно-технологического развития Российской Федерации: Указ Президента Российской Федерации от 01.12.2016 г. № 642 // Собрание законодательства РФ. 05.12.2016. № 49 (ч. І). Ст. 6887. 
(СППР) при осуществлении технологических процессов. В сфере добычи углеводородного сырья такие системы высоко востребованы в процессах обслуживания и управления нефтегазодобывающими скважинами, нефтепроводами и другими технологическими объектами.

\section{1. Анализ предметной области}

В процессе добычи нефти используется большое количество информационных систем, данные из которых надо учитывать при обслуживании технологического оборудования:

- гидродинамические модели месторождений и цифровые модели добывающих скважин позволяют рассчитать оптимальный режим работы скважин;

- системы автоматизированного управления (АСУТП) позволяют удаленно собирать текущие значения параметров работы оборудования и изменять режимы работы;

- специализированные хранилища данных позволяют хранить историю изменения значений параметров большого количества установок в течение длительного промежутка времени, быстро обращаться к необходимым данным;

- системы планирования ремонтов (ТОРО) позволяют хранить историю ремонтов оборудования, планировать будущие ремонты и закупку необходимых комплектующих.

Другой особенностью процесса обслуживания нефтедобывающих скважин является высокая территориальная распределенность оборудования. Требование принятия решений по обслуживанию далеких от центральной инфраструктуры объектов, где зачастую отсутствуют все дешевые виды связи, влечет необходимость реализации СППР в виде мобильного приложения для операторов-обходчиков с возможностью работы без соединения с серверами. При этом вся работа со справочниками и интеграция с другими информационными системами должна осуществляться централизованно, поэтому для СППР требуется специальный сервер, обмен данными с которым будет происходить в начале и в конце обходов оборудования.

Таким образом, диаграмма укрупненных потоков данных в такой СППР может выглядеть следующим образом (рис. 1).

Наиболее массовыми видами применяемого насосного оборудования являются центробежный (ЭЦН) и штанговый глубинный (ШГН) 
насосы [1]. Основными параметрами работы этих насосов являются: нагрузка, загрузка, частота двигателя; буферное, линейное, затрубное давления; температура, сопротивление изоляции, вибрация, расход жидкости. Часть параметров может быть измерена автоматически и оцифрована, часть собирается вручную.

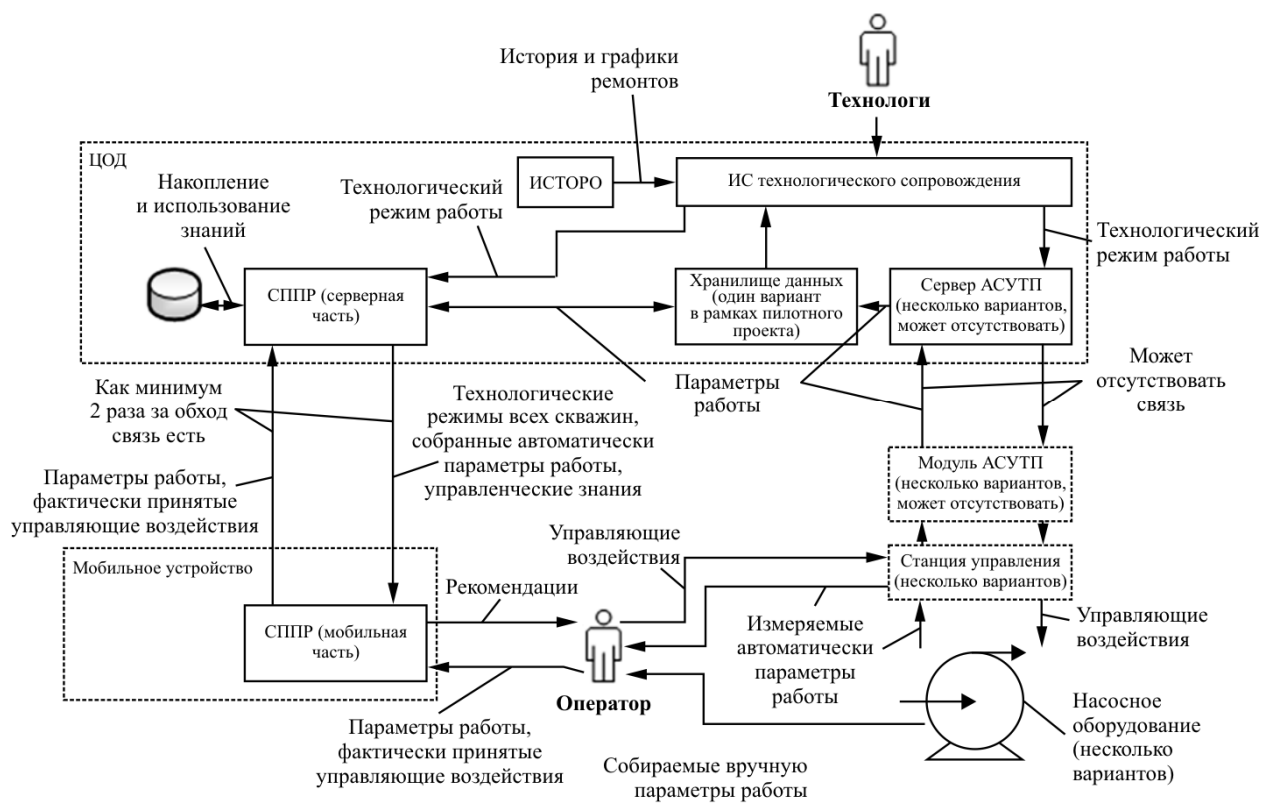

Рис. 1. Информационные системы и основные потоки данных в процессе обслуживания технологического оборудования добычи нефти (авторские результаты)

Наиболее частыми операциями по обслуживанию являются: скребкование, опрессовка труб, встряхивание изменением частоты и вращения, пропарка и ревизия нефтепровода и измерительных устройств, чистка и замена штуцера, поиск прорывов в нефтепроводе. Часть операций не может быть выполнена операторами лично и требует вызова специальных сервисных бригад.

Основные входные и выходные информационные потоки при расчете рекомендаций показаны на схеме (рис. 2).

Построение автоматизированной интеллектуальной системы поддержки принятия решений с учетом этих требований может быть осуществлено, например, с использованием нейронных сетей, лесов деревьев решений, нечеткой логики или алгоритмов классификации. 
Для обучения этих моделей необходимо в цифровом виде получить базу знаний или обучающую выборку, в которой должна быть зафиксирована связь между значениями параметров работы оборудования и совершенными ранее или необходимыми действиями операторов по обслуживанию технологического оборудования. Источниками таких данных могут стать существующие информационные системы, при наличии доступа к их архивам:

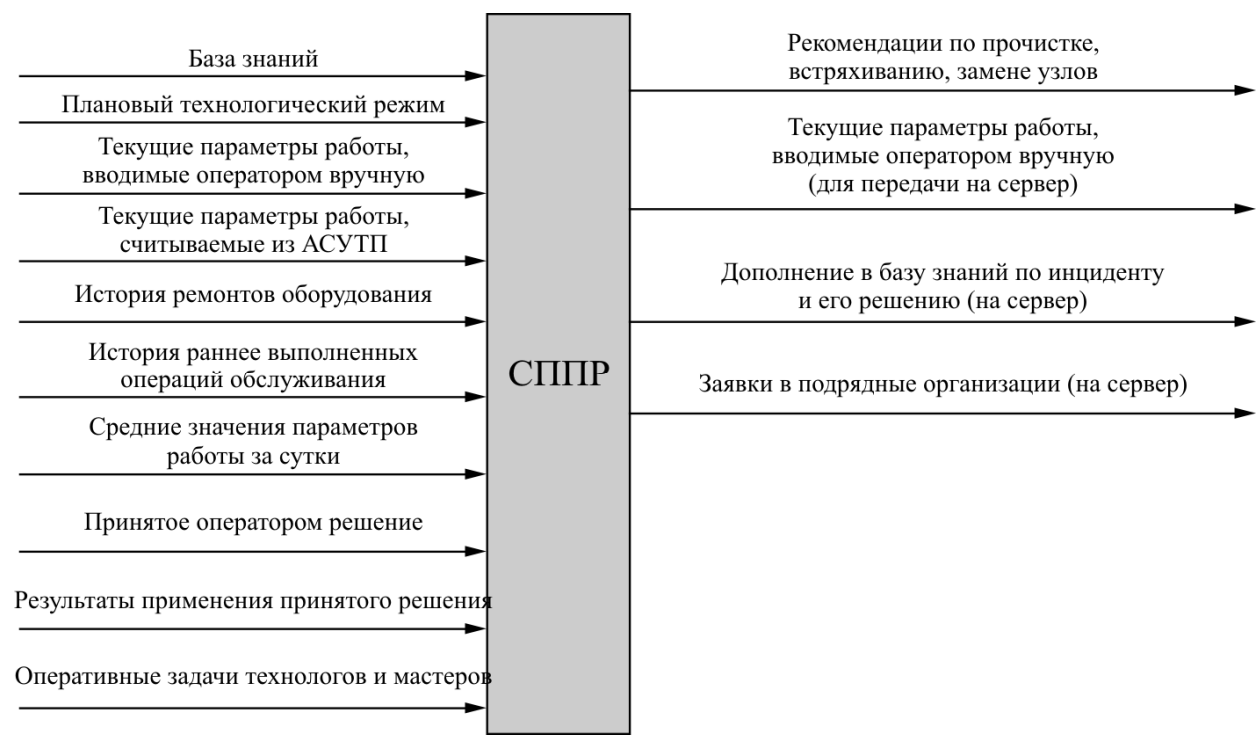

Рис. 2. Описание СППР в нотации «черный ящик» (авторские результаты)

- в системах ТОРО хранятся фактические данные по выполнению ремонтных работ, но не всех возможных операций обслуживания;

- гидродинамические модели месторождений и модели скважин позволяют рассчитать оптимальный плановый режим работы (частоту двигателя, глубину спуска насоса) и получить его историю;

- системы АСУТП и хранилища данных при наличии связи позволяют получить архив значений измеряемых и оцифрованных параметров работы скважин.

Но история значений части параметров работы, которые не поддаются оцифровке или автоматический сбор которых на текущий момент является нецелесообразным, а также фактически выполненные действия операторов, не связанные с ремонтами, фиксируются посуточно только в бумажных журналах. 
Оцифровка достаточного количества журналов для подготовки репрезентативной обучающей выборки может оказаться весьма трудоемкой задачей. Генерация синтетической обучающей выборки потребует одновременно частичной оцифровки некоторых журналов, знаний технологов, разработки вспомогательного генератора и верификации полученных данных.

Таким образом, нейронные сети или деревья решений, требующие для обучения числовую оцифрованную выборку, не подходят для решения поставленной задачи с учетом обозначенных ограничений.

В случае, когда принятие решений по обслуживанию оборудования осуществляется людьми - экспертами из службы технологов, наименее ресурсозатратным способом получения знаний может быть интервью технологов. Результатом такого интервью является база знаний, состоящая из ограниченного набора правил вида «если ..., то ...», которые легко могут быть обработаны с помощью аппарата нечеткой логики.

Нечеткая логика и ее методы нашли многочисленные применения в задачах моделирования и управления [2-4]. Для численной обработки экспертных правил в ней используются нечеткие множества, а логические операции над нечеткими множествами моделируются, например, с помощью предложенных Л. Заде нечетких конъюнкции $x \wedge y=\min (x, y)$ и дизъюнкции $x \vee y=\max (x, y)$ [5].

Преимущества использования нечеткой логики для решения поставленной задачи следующие:

- малые трудозатраты на получение знаний;

- обоснование явным образом получаемых результатов (какие конкретно правила привели к получению результата);

- возможность проверки полноты БЗ на уровне лингвистических правил.

Трудности использования нечеткой логики следующие:

- построение объективных функций принадлежности/истинности;

- выбор подходящих моделей логических операций;

- проверка полноты и непротиворечивости БЗ на уровне функций истинности;

- автоматическая корректировка знаний в процессе эксплуатации системы. 


\section{2. Матричный аппарат нечеткой логики}

Для решения указанных трудностей предлагается применить матричный аппарат нечеткой логики [6-8]. В нем используются модели логических операций, естественным образом обобщающие их «четкие» аналоги на область нечетких переменных и предикатов. Этот аппарат позволяет свести задачи нечеткого логического вывода к решению системы линейных алгебраических уравнений с известными условиями существования и единственности решений.

\section{3. Векторное представление нечетких высказываний и переменных}

Под высказыванием $A$ в обычной (четкой) логике понимают такое предложение, которое либо истинно, либо ложно. На совокупности всех высказываний можно определить функцию $\mu(A)$, принимающую значения в двухэлементном множестве $\{0,1\}$, по следующему правилу:

$$
\mu(A)=\left\{\begin{array}{c}
1, \text { если высказывание } A \text { истинно, } \\
0, \text { если высказывание } A \text { ложно. }
\end{array}\right.
$$

Функция $\mu(A)$ называется функцией истинности, а ее значения логическим значением или значением истинности высказывания $A$.

Под высказыванием в нечеткой логике понимается такое предложение, которое может принимать истинное значение с какой-то степенью уверенности. На совокупности нечетких высказываний можно определить функцию истинности $\mu(A)$, принимающую значения на отрезке $[0,1]$, равную степени уверенности в справедливости соответствующего высказывания.

В матричном представлении [6] для описания степени истинности высказывания $A$ вводится двухмерный вектор х. Если ввести базисные векторы $\mathbf{e}^{(0)}$ и $\mathbf{e}^{(1)}$, то вектор $\mathbf{x}$ может быть представлен в виде

$$
\mathbf{x}=x_{0} \mathbf{e}^{(0)}+x_{1} \mathbf{e}^{(1)},
$$

причем компоненты вектора $\mathbf{x}$ - числа $x_{0}, x_{1}$ - удовлетворяют условиям

$$
0 \leq x_{0}, x_{1} \leq 1, x_{0}+x_{1}=1 .
$$


Сами базисные векторы $\mathbf{e}^{(0)}$ и $\mathbf{e}^{(1)}$ описывают высказывания со степенью уверенности 0 и 1 соответственно. Операции отрицания соответствует перестановка компонент вектора $\mathbf{x}: \neg \mathbf{x}=x_{1} \mathbf{e}^{(0)}+x_{0} \mathbf{e}^{(1)}$.

Логические операции над векторами - конъюнкцию, дизъюнкцию и импликацию - можно представить в матричном виде. Составим из компонент вектора $\mathbf{x}$ конъюнктивную $\mathbf{C}(\mathbf{x})$, дизъюнктивную $\mathbf{D}(\mathbf{x})$ и импликативную $\mathbf{I}(\mathbf{x})$ матрицы размером $2 \times 2$ [3]:

$$
\mathbf{C}(\mathbf{x})=\left(\begin{array}{ll}
1 & x_{0} \\
0 & x_{1}
\end{array}\right) ; \mathbf{D}(\mathbf{x})=\left(\begin{array}{ll}
x_{0} & 0 \\
x_{1} & 1
\end{array}\right) ; \mathbf{I}(\mathbf{x})=\left(\begin{array}{ll}
x_{1} & 0 \\
x_{0} & 1
\end{array}\right) .
$$

С помощью этих матриц логические операции над нечеткими векторами могут быть представлены в виде

$$
\mathbf{x} \wedge \mathbf{y}=\mathbf{C}(\mathbf{x}) \cdot \mathbf{y} ; \quad \mathbf{x} \vee \mathbf{y}=\mathbf{D}(\mathbf{x}) \cdot \mathbf{y} ; \mathbf{x} \rightarrow \mathbf{y}=\mathbf{I}(\mathbf{x}) \cdot \mathbf{y},
$$

где вектор у следует мыслить как матричный столбец $1 \times 2$, составленный из его компонент $\mathbf{y}=\left(\begin{array}{l}y_{0} \\ y_{1}\end{array}\right)$, а точка обозначает обычное матричное умножение. Результатом логических операций, сформулированных в таком виде, тоже будет нечеткий вектор. Детальное обоснование сделанного выбора матриц (4) представлено в работе [6].

\section{4. Нечеткие векторные предикаты}

В классическом подходе [5, 9] нечеткая переменная характеризуется нечетким подмножеством $X$ некоторого универсального множества $U$ с функцией принадлежности $\mu_{x}(u), u \in U$. Например, переменная «дебит жидкости большой» может характеризоваться функцией принадлежности, изображенной на рис. 3 красным цветом.

Значение функции принадлежности может выступать как свойство некоторого объекта из множества $U$. Такое описание хорошо подходит под определение предикатов, форма представления которых предложена в работе [6].

Нечеткий предикат $\mathbf{x}(u)$ - это векторная функция, заданная на множестве определения $U$, принимающая значения в пространстве нечетких векторов $F$, элементы которого описываются формулами (2) и (3), т.e. 


$$
\begin{gathered}
\mathbf{x}(u)=\left(\begin{array}{c}
x_{0}(u) \\
x_{1}(u)
\end{array}\right) ; \\
0 \leq x_{0}(u), x_{1}(u) \leq 1 ; x_{0}(u)+x_{1}(u)=1 .
\end{gathered}
$$

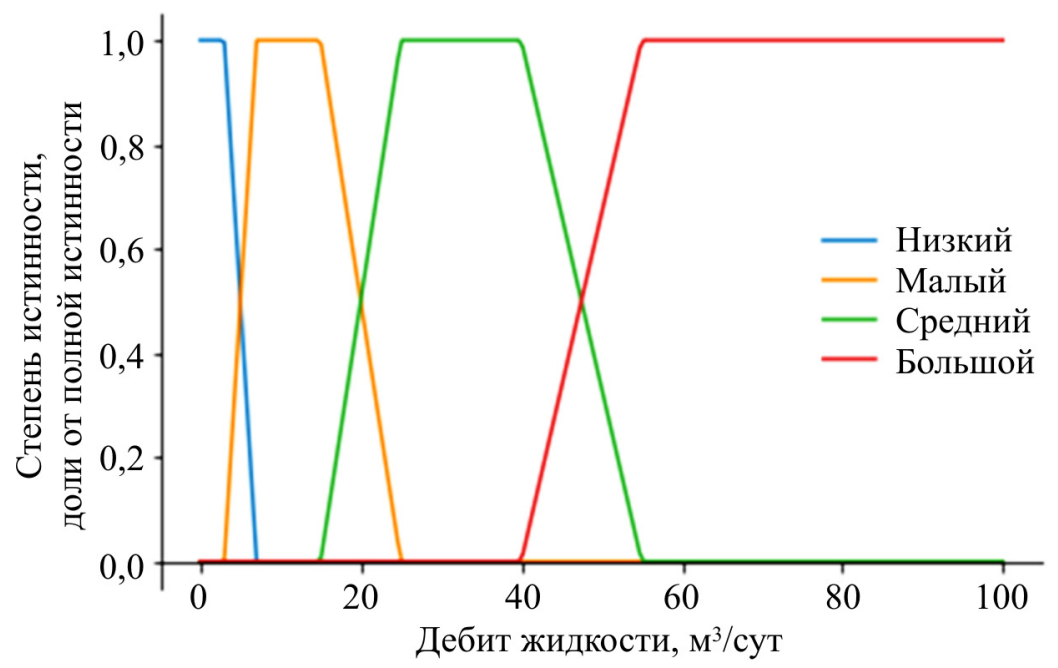

Рис. 3. Внешний вид функций принадлежности нечетких множеств, описывающих высказывания о размере суточного дебита жидкости

Из определения предиката следует, что в качестве значения $x_{1}(u)$ используется значение функции принадлежности нечеткой переменной $\mu_{x}(u)$, в то время как для выполнения условий (3) необходимо $x_{0}(u)=1-\mu_{x}(u)$. Операции над нечеткими предикатами сводятся к операциям над нечеткими векторами.

\section{5. Нечеткий логический вывод}

Нечеткий логический вывод, или приближенные рассуждения это процесс получения значений искомых нечетких переменных или предикатов по заданным значениям исходных переменных с использованием логических правил modus ponens вида «если ..., то ...», modus tollens и др. Предпосылки и выводы правил являются нечеткими понятиями. Совокупность всех правил представляет собой базу знаний, которая формируется человеком - экспертом в данной предметной области. Общие этапы логического вывода, а также примеры реализаций 
в «классической» формулировке нечеткой логики по алгоритмам Mamdani, Larsen и другим описаны во многих источниках (см., например, работы [2, 9]). В этом подходе предлагается задавать правила в базе знаний с помощью отношений $x \rightarrow y=\mathbf{R}$, где матрица $\mathbf{R}$ должна быть эмпирически задана на множестве $x \times y$ (здесь знак «х» обозначает прямое произведение нечетких переменных $x, y$ ). Обобщение правила modus ponens на нечеткую логику, наиболее точно соответствующее операции импликации в четкой логике, предложено в работе [6]. Оно имеет аналогичное четкому варианту поведение в особых точках и учитывает факт связи между предпосылкой, следствием и степенью выполнения правила.

Если известен нечеткий вектор $\mathbf{x}=\left(\begin{array}{l}x_{0} \\ x_{1}\end{array}\right)$, а также нечеткий вектор $\mathbf{z}$, определяющий нечеткость правила $\mathbf{x} \rightarrow \mathbf{y}=\mathbf{z}$, то вывод о нечеткости вектора $\mathbf{y}$ можно сделать из соотношений (4). Эта задача сводится к решению линейного алгебраического уравнения относительно неизвестного вектора $\mathbf{y}$, которое в случае импликативной модели имеет вид $\mathbf{I}(\mathbf{x}) \cdot \mathbf{y}=\mathbf{z}$ :

$$
\left(\begin{array}{ll}
x_{1} & 0 \\
x_{0} & 1
\end{array}\right)\left(\begin{array}{l}
y_{0} \\
y_{1}
\end{array}\right)=\left(\begin{array}{c}
z_{0} \\
z_{1}
\end{array}\right) \text { или }\left\{\begin{array}{c}
x_{1} y_{0}=z_{0} \\
x_{0} y_{0}+y_{1}=z_{1}
\end{array}\right.
$$

Как известно, линейная система имеет решение, если определитель матрицы коэффициентов не равен нулю. В данном случае мы должны потребовать, чтобы выполнялось условие

$$
\operatorname{det}(\mathbf{I}(\mathbf{x}))=\operatorname{det}\left(\begin{array}{ll}
x_{1} & 0 \\
x_{0} & 1
\end{array}\right)=x_{1} \neq 0
$$

Иными словами, компонента истинности $x_{1}$ вектора $\mathbf{x}$ не должна быть равна нулю. Тогда компоненты неизвестного вектора $\mathbf{y}$ принимают значения

$$
y_{0}=\frac{z_{0}}{x_{1}} ; \quad y_{1}=1-\frac{z_{0}}{x_{1}}
$$


Полученное решение имеет смысл, только если обе компоненты вектора у соответствуют условиям (3), поэтому, кроме условия (9), получаем дополнительно, что $x_{1} \geq z_{0}$. Отметим, что при $x_{1}=1$ и $z_{1}=1$ из формул (10) имеем известный результат правила modus ponens в четкой логике: $y_{0}=0 ; y_{1}=1$. Таким образом, в отличие от других моделей импликации, в этом подходе мы не только получаем нечеткий логический вывод, но и строго указываем область его выполнения. Аналогичным образом могут быть обобщены на область нечетких переменных и другие известные правила логического вывода [6].

\section{6. Матричная реализация нечеткого логического вывода с использованием нечетких предикатов}

В большинстве практических задач управления недостаточно вывода на основе отдельных высказываний, так как все правила формулируются на языке лингвистических переменных. В связи с этим предложено обобщение матричной реализации нечеткого вывода на область нечетких множеств, предикатов и лингвистических переменных.

В качестве исходных данных используется база знаний, состоящая из правил вида «если ..., то...», сформулированных на естественном языке, т.е. с использованием значений лингвистических переменных $\alpha$ и $\gamma$, описывающих входной и выходной параметры соответственно. Для каждого правила должна быть указана степень истинности $\mathbf{z}$. Кроме того, должны быть установлены допустимые диапазоны значений входного $u$ и искомого $v$ параметров задачи (универсальные множества $U$ и $V$ ), заданы фактические четкие значения входного параметра $u_{0}$.

Лингвистические правила условно можно записать в виде зависимостей между значениями лингвистических переменных $\alpha$ и $\gamma$, а также степенью истинности правил

$$
\gamma_{k}=\Gamma\left(\alpha_{i}, \mathbf{z}_{i, k}\right)
$$

где используются следующие лингвистические переменные:

$\alpha=\left\{\mathbf{x}_{\text {мальй }}(u), \ldots, \mathbf{x}_{\text {большой }}(u)\right\}-$ характеризует входной параметр $u \in U$; 
$\gamma=\left\{\mathbf{y}_{\text {малый }}(v), \ldots, \mathbf{y}_{\text {большой }}(v)\right\}-$ характеризует результат $v \in V$.

Каждое правило имеет степень истинности $\mathbf{z}_{i, k}$. В общем случае матрица степеней нечеткости может быть многомерной. В случае, если нет правила, устанавливающего зависимость между значением входного параметра $\alpha_{i}$ и значением результата $\gamma_{k}$, соответствующее значение $\mathbf{z}_{i, k}$ равно $\mathbf{z}_{i, k}=\left(\begin{array}{l}1 \\ 0\end{array}\right)$.

С помощью логических операций правила записываются следующим образом:

$$
\alpha_{i} \rightarrow \gamma_{k}=\mathbf{z}_{i, k}
$$

Задача состоит в том, чтобы численно рассчитать значения параметра $v_{0} \in V$ по заданному значению параметра $u_{0} \in U$.

В первую очередь приводим четкое значение $u_{0}$ к нечеткому виду с использованием функций принадлежности $\mu_{\alpha i}(u)$ нечетких множеств, формализующих все возможные значения $\alpha_{i}$ лингвистической переменной $\alpha$. Другими словами, необходимо найти значения нечетких предикатов $\mathbf{x}_{i}\left(u_{0}\right)$, задающихся функциями истинности $\mu_{\alpha i}(u)$.

Правила (12) в матричной реализации операций над предикатами перепишутся следующим образом:

$$
\mathbf{I}\left(\mathbf{x}_{i}\left(u_{0}\right)\right) \cdot \widetilde{\mathbf{y}_{k}}=\mathbf{z}_{i, k}
$$

где $\widetilde{\mathbf{y}_{k}}-$ степень фактической реализации правила с номером $k$, или степень истинности высказывания; $\gamma_{k}$ - следствия данного правила. Решение этого уравнения имеет вид

$$
\begin{gathered}
\widetilde{\mathbf{y}_{k}}=\left(\mathbf{I}^{-1}\left(\mathbf{x}_{0}\left(u_{0}\right)\right) \cdot \mathbf{z}_{0, k}\right) \vee\left(\mathbf{I}^{-1}\left(\mathbf{x}_{1}\left(u_{0}\right)\right) \cdot \mathbf{z}_{1, k}\right) \vee \ldots= \\
=D \ldots\left(D\left(\mathbf{I}^{-1}\left(\mathbf{x}_{0}\left(u_{0}\right)\right) \cdot \mathbf{z}_{0, k}\right) \cdot\left(\mathbf{I}^{-1}\left(\mathbf{x}_{1}\left(u_{0}\right)\right) \cdot \mathbf{z}_{1, k}\right)\right) \cdot \ldots= \\
=\underset{\text { all } i}{D}\left(\mathbf{I}^{-1}\left(\mathbf{x}_{i}\left(u_{0}\right)\right) \cdot \mathbf{z}_{i, k}\right),
\end{gathered}
$$


где введено обозначение $\underset{\text { all } i}{D}$ - дизъюнкция нечетких векторов по всем значениям индекса $i$, которая возможна благодаря сохранению ассоциативных и дистрибутивных свойств.

Чтобы найти четкое значение результирующего параметра, строят вспомогательную функцию

$$
\mathbf{r}(v)=\underset{\text { all } k}{D}\left\{C\left[\widetilde{\mathbf{y}_{k}}\right] \mathbf{y}_{k}(v)\right\}
$$

В конце производится дефаззификация:

$$
v_{0}=\frac{\int_{V} v \cdot \mathbf{r}_{1}(v) d v}{\int_{V} \mathbf{r}_{1}(v) d v}
$$

Необходимо отметить, что, как было продемонстрировано выше, формула (14) не всегда дает результат, удовлетворяющий условиям (3). Это означает, что данное правило не может характеризовать величину искомого параметра $v_{0}$ при данном значении входного параметра $u_{0}$. На практике в таком случае можно установить значение $\widetilde{\mathbf{y}_{k}}=\left(\begin{array}{l}1 \\ 0\end{array}\right)$ либо сообщить оператору о неполноте базы знаний.

\section{7. Нечеткий логический автомат с памятью}

Описанный выше алгоритм нечеткого вывода можно рассматривать как комбинационную логику или алгоритм принятия решений на основании информации о текущем состоянии объекта. Для анализа истории изменения параметров объекта (ретроспективной информации), как требуется в процессе обслуживания технологического оборудования нефтедобычи, популярным решением является применение логических автоматов с памятью. При этом для одновременнго использования знаний экспертов требуется применение нечеткого автомата с памятью [10, 11].

Однако при конкретной реализации таких автоматов встречаются трудности, связанные с тем, что «память» автомата задается неявно с помощью введения «состояний» автомата и переходов между ними. Ввиду этого при решении задач необходимо делать выбор оптимально- 
го числа состояний, как это обсуждается, например, в работе [12]. Кроме того, сложность вызывает построение функций истинности нечетких состояний.

Для решения этой проблемы предлагается новая модель нечеткого автомата с памятью в виде нечеткой комбинационной схемы, анализирующей содержимое внешнего блока памяти. При этом результат работы такой модели должен соответствовать результатам работы нечеткого автомата. Для построения этой модели проанализируем алгоритмы работы четких автоматов и комбинационных схем, а также нечетких автоматов в их обычном представлении.

\section{8. Представление «четкого» автомата в виде модифицированной комбинационной схемы}

Как известно, логические («четкие») автоматы с памятью (см., например, [13; 14]) описываются кортежем $\{X, Y, S, F, G\}$, где $X$ - набор возможных значений (алфавит) входных переменных, $Y$ - алфавит выходных сигналов, $S$ - набор состояний автомата, а $F$ и $G$ - функция переходов и функция выходов соответственно: $s_{t+\Delta t}=F\left(s_{t}, x_{t}\right)$, $y_{t}=G\left(s_{t}, x_{t}\right)$, где $x_{t} \in X, y_{t} \in Y, s_{t} \in S-$ конкретные значения входных параметров, состояний и выходов в момент времени $t$. Для конечных автоматов каждый из алфавитов $X, Y, S$ является конечным.

В конкретных приложениях используются автоматы с конечной памятью (АКП), понятие о которых приведено в работе Брауэра [13]. Работа таких автоматов оказывается эквивалентной отображению вида

$$
y_{t}=h\left(x_{t-p \Delta t}, \ldots, x_{t-\Delta t}, x_{t}, y_{t-q \Delta t}, \ldots, y_{t-2 \Delta t}, y_{t-\Delta t}\right),
$$

где $x_{t-p \Delta t}, \ldots, x_{t-\Delta t}, x_{t}-$ значения входных переменных; $y_{t-q \Delta t}, \ldots, y_{t-2 \Delta t}, y_{t-\Delta t}-$ значения выходных переменных; $p$ и $q$ - конечные положительные целые числа, $q<p$. Легко видеть, что отображение (17) для конечного автомата может быть реализовано с помощью комбинационной схемы $(К С)$, анализирующей внешний блок памяти (ВБП). В текущий момент времени $t$ в ВБП хранится совокупность аргументов отображения (17) $x_{t-p \Delta t}, \ldots, x_{t-\Delta t}, x_{t}, y_{t-q \Delta t}, \ldots, y_{t-2 \Delta t}, y_{t-\Delta t}$, а роль комбинационной схемы состоит в том, чтобы в зависимости от содержания ВБП определить зна- 
чение выходной переменной $y_{t}$ в соответствии с формулой (17). Далее мы ограничиваемся более простым, но практически важным случаем, когда отображение (17) имеет вид формулы (18). Применение развиваемого подхода для более общего случая, соответствующего зависимости (17), не представляет особых затруднений.

$$
y_{t}=h\left(x_{t-p \Delta t}, \ldots, x_{t-\Delta t}, x_{t}\right) .
$$

Это значит, что для работы КС в блоке памяти достаточно сохранять значения только входных переменных в текущий момент времени $t$ и в предшествующие моменты времени $t-\Delta t>\ldots>t-p \Delta t$. Далее параметр $p$ будем называть «глубиной» памяти входных переменных. Содержание памяти обновляется на каждом шаге по алгоритму FIFO (First Input, First Output - первый вошел, первый вышел).

Комбинационная схема анализирует набор значений входных переменных $x_{t-p \Delta t}, \ldots, x_{t-\Delta t}, x_{t}$ и сопоставляет ему определенное значение выходной переменной $y_{t}$.

Таким образом, если память четкого логического автомата конечна, то можно поставить в соответствие ему четкую комбинационную схему, анализирующую внешний блок памяти. Пример такого сопоставления приведен в работе [15].

\section{9. Алгоритмическая модель нечеткого автомата}

Будем исходить из того, что в основе модели нечеткого автомата с конечной памятью (НАКП) лежит некоторый четкий автомат с конечной памятью (АКП). Для описания этого АКП вводятся переменные $x, y, s$, принимающие значения на конечных множествах $A, B, C$ $(x \in A, y \in B, s \in C)$. Для АКП также считаются известными автоматные функции $s_{t+\Delta t}=F\left(s_{t}, x_{t}\right), y_{t}=G\left(s_{t}, x_{t}\right)$, которые могут быть заданы табличным способом. Далее, при переходе к модели нечеткого автомата, множества $A, B, C$ мы будем считать «универсальными» множествами, на которых будут задаваться нечеткие подмножества, описывающие лингвистические переменные.

Для описания НАКП введем «лингвистические» переменные: $\alpha, \beta, \gamma$, где $\alpha, \beta$ - входная и выходная переменные, а $\gamma$ - переменная 
состояния. Каждая из величин $\alpha, \beta$ и $\gamma$ принимает значения из наборов $\left\{\alpha_{0}, \alpha_{1}, \ldots, \alpha_{u}\right\},\left\{\beta_{0}, \beta_{1}, \ldots, \beta_{v}\right\},\left\{\gamma_{0}, \gamma_{1}, \ldots, \gamma_{w}\right\}$ соответственно. Величины $\alpha_{i}, i \in[0, u], \beta_{j}, j \in[0, v], \quad \gamma_{k}, k \in[0, w]$ количественно описываются нечеткими подмножествами введенных выше универсумов $A, B, C$ соответственно с помощью функций принадлежности $\mu_{\alpha i}(x)$, $\mu_{\beta j}(y)$ или $\mu_{\gamma k}(s)$. Далее мы будем отождествлять лингвистические переменные и описывающие их нечеткие множества.

Алгоритм работы нечеткого автомата формулируется на языке лингвистических переменных. Переходы между состояниями автомата и значение выходного сигнала выражаются нечеткими автоматными функциями

$$
\gamma_{t+\Delta t}=\Gamma\left(\alpha_{t}, \gamma_{t}\right), \beta_{t}=\mathrm{B}\left(\alpha_{t}, \gamma_{t}\right)
$$

Как и в случае четкого конечного автомата, функции Г и В могут быть заданы табличным методом. Эти таблицы совпадают с аналогичными таблицами автоматных функций четкого автомата, но с тем отличием, что как в заголовки таблиц, так и в их ячейки должны быть подставлены соответствующие лингвистические переменные. Автоматные функции НАКП могут быть заданы и с помощью более кратких индексных обозначений:

$$
\sum_{\text {all } i, n} \boldsymbol{\varepsilon}_{i, n, m} \alpha_{i} \wedge \gamma_{n} \rightarrow \gamma_{m} ; \quad \sum_{\text {all } i, n} \boldsymbol{\delta}_{i, n, j} \alpha_{i} \wedge \gamma_{n} \rightarrow \beta_{j},
$$

где введены матрицы переходов $\boldsymbol{\varepsilon}_{i, n, m}$ и выходов $\boldsymbol{\delta}_{i, n, j}$ автомата, компоненты которых принимают значения, равные 0 или 1 в соответствии с заданными конкретными правилами «если ..., то...». В таком же виде могут быть выражены автоматные функции и АКП. Логические связки $\wedge, \rightarrow$ между нечеткими множествами могут быть реализованы как по известным правилам, введенным Заде [5], так и нечетким логическим выводом в матричной реализации. В случае автомата Мура выходной сигнал определяется только текущим состоянием автомата и правила (20) сводятся к следующему виду:

$$
\sum_{\text {all } i, n} \boldsymbol{\varepsilon}_{i, n, m} \alpha_{i} \wedge \gamma_{n} \rightarrow \gamma_{m} ; \quad \sum_{\text {all } n} \boldsymbol{\delta}_{n, j} \gamma_{n} \rightarrow \beta_{j}
$$


Отметим, что состояния нечеткого автомата описываются с помощью лингвистической переменной $\gamma$. Значения этой переменной задаются нечеткими подмножествами универсального множества состояний $C$, включающего все состояния АКП, и функциями принадлежности $\mu_{\gamma k}(s)$. Ввиду этого можно сказать, что у НАКП с разной степенью активны сразу несколько состояний соответствующего ему АКП, причем степень активности определяется функцией $\mu_{\gamma k}(s)$.

Для численной реализации работы НКАП будем считать, что в текущий момент времени $t$ известны параметры $x_{t}, s_{t}$. Практически входные и выходные параметры нечеткого автомата (как и у четкого) задаются некоторыми «четкими» числами. Например, на входе автомата это показания датчиков, а на выходе номер принимаемого решения из списка по обслуживанию оборудования. Отличие нечеткого автомата от «четкого» заключается в использовании другого алгоритма преобразования входного сигнала в выходной.

Вычислительная процедура строится следующим образом. Для заданных значений $x_{t}, s_{t}$ с помощью функций $\mu_{\alpha i}\left(x_{t}\right), \mu_{\gamma k}\left(s_{t}\right)$ определяем их степени принадлежности нечетким множествам $\alpha_{i}, \gamma_{j}$ (либо степени истинности векторных предикатов в матричном представлении), которые представляют собой некоторые наборы чисел, лежащих на отрезке $[0,1]$. Для вычисления величин $y_{t+\Delta t}, s_{t+\Delta t}$ воспользуемся матричным алгоритмом нечеткого вывода. По аналогии с формулой (15) введем вспомогательные функции $\mathbf{Q}(s), \mathbf{R}(y)$ :

$$
\begin{aligned}
& \mathbf{Q}(s)=D_{i, n, m} \boldsymbol{\varepsilon}_{i, n, m} \cdot C\left(\mathbf{I}^{-1}\left(C\left(\mathbf{x}_{\alpha i}\left(x_{t}\right)\right) \cdot \mathbf{s}_{\gamma n}\left(s_{t}\right)\right) \cdot \mathbf{z}_{i n m}\right) \cdot \mathbf{s}_{\gamma m}(s) ; \\
& \mathbf{R}(y)=D_{i, n, j} \boldsymbol{\delta}_{i, n, j} C\left(\mathbf{I}^{-1}\left(C\left(\mathbf{x}_{\alpha i}\left(x_{t}\right)\right) \cdot \mathbf{s}_{\gamma n}\left(s_{t}\right)\right) \cdot \mathbf{z}_{i n j}\right) \cdot \mathbf{y}_{\beta j}(y),
\end{aligned}
$$

где использованы нечеткие векторные предикаты

$$
\mathbf{x}_{\alpha i}(x)=\left(\begin{array}{c}
1-\mu_{\alpha i}(x) \\
\mu_{\alpha i}(x)
\end{array}\right), \mathbf{s}_{\gamma k}(s)=\left(\begin{array}{c}
1-\mu_{\gamma k}(s) \\
\mu_{\gamma k}(s)
\end{array}\right), \mathbf{y}_{\beta j}(y)=\left(\begin{array}{c}
1-\mu_{\beta j}(y) \\
\mu_{\beta j}(y)
\end{array}\right)
$$


С помощью этих функций производим дефаззификцию:

$$
\bar{s}=\frac{\int_{\text {all } S} s \mathbf{Q}(s)_{1} d s}{\int_{\text {all } S} \mathbf{Q}(s)_{1} d s} ; \bar{y}=\frac{\int_{\text {all } y} y \mathbf{R}(y)_{1} d y}{\int_{\text {all } y} \mathbf{R}(y)_{1} d y} .
$$

На этом завершается обработка значения входного сигнала, и автомат ожидает поступление на вход следующего сигнала.

\section{0. Модифицированная нечеткая комбинационная схема}

Алгоритм работы МНКС определяется набором правил, сформулированных экспертом на языке лингвистических переменных $\alpha$ и $\beta$, соответствующих входным и выходным параметрам (введение лингвистической переменной состояний здесь не требуется). Они имеют значения $\alpha_{0}, \alpha_{1}, \ldots \alpha_{u}, \beta_{0}, \beta_{1}, \ldots \beta_{v}$, которые количественно описываются функциями $\mu_{\alpha i}(x)$ и $\mu_{\beta j}(y)$ (пример функций см. на рис. 3 ). Входные и выходные параметры принимают значения на универсальных множествах $A, B$, так что $x \in A, y \in B$. В момент времени $t$ на вход комбинационной схемы подается последовательность значений входного параметра $\alpha_{t-p \Delta t}, \ldots, \alpha_{t-\Delta t}, \alpha_{t}$ за некоторый промежуток времени $(p+1) \Delta t$. Значение выходного сигнала задается некоторой функцией $\beta_{t}=H\left(\alpha_{t-p \Delta t}, \ldots, \alpha_{t-\Delta t}, \alpha_{t}\right)$. Для дальнейшего использования перепишем ее в виде индексной записи:

$$
\beta_{j}=H\left(\alpha_{i p}, \ldots \alpha_{i 1}, \alpha_{i 0}\right) .
$$

Функциональная зависимость $H$ задается правилами вида «если ..., то ...» и в терминах логических операций над нечеткими множествами может быть сформулирована в индексной записи следующим образом:

$$
\sum_{\text {all } i p, \ldots, i 1, i 0} \boldsymbol{\delta}_{i p, \ldots, i 1, i 0, j} \alpha_{i p} \wedge \ldots \wedge \alpha_{i 1} \wedge \alpha_{i 0} \rightarrow \beta_{j}
$$

Значения компонент матрицы выходного сигнала $\boldsymbol{\delta}_{i p, \ldots, i 1, i 0, j}$ определяются заданными правилами «если ..., то ...». 
Количественная реализация МНКС сводится к нахождению параметра $y_{t}$ по заданной последовательности значений входного параметра $x_{t-p \Delta t}, \ldots, x_{t-\Delta t}, x_{t}$. По этим величинам входного параметра находим значения функций принадлежности для всех значений входной лингвистической переменной $\alpha \mu_{\alpha i p}\left(x_{t-p \Delta t}\right), \ldots, \mu_{\alpha i 0}\left(x_{t-\Delta t}\right), \mu_{\alpha i 0}\left(x_{t}\right)$, которые являются некоторыми числами из отрезка $[0,1]$. Для вычисления $y_{t}$ в соответствии с матричной реализацией нечеткого вывода строим вспомогательную функцию $\mathbf{V}(y)$, имеющую следующий вид:

$$
\mathbf{V}(y)=\underset{i p, \ldots, i 0, j}{D} \boldsymbol{\delta}_{i p, \ldots, i 0, j} C\left(\mathbf{I}^{-1}\left(C\left(\mathbf{x}_{\alpha i p}\left(x_{t-p \Delta t}\right), \ldots, \mathbf{x}_{\alpha i 0}\left(x_{t}\right)\right)\right) \mathbf{z}_{i p, \ldots, i 0, j}\right) \mathbf{y}_{\beta j}(y)
$$

С помощью этой функции производим дефаззификацию:

$$
\bar{y}=\frac{\int_{\text {all } y} y \mathbf{V}(y)_{1} d y}{\int_{\text {all } y} \mathbf{V}(y)_{1} d y} .
$$

На этом завершается обработка очередного значения входного сигнала, и МНКС ожидает поступление на вход следующего сигнала.

\section{1. Построение интеллектуальной системы поддержки принятия решений}

Описанный выше математический аппарат позволяет строить интеллектуальные системы поддержки принятия решений, учитывающие одновременно и историю изменения параметров объекта, и лингвистические экспертные правила формирования решений.

Для определения перечня функций информационной системы построены детальные модели бизнес-процессов нижнего уровня, выполняемые в рамках обслуживания технологического оборудования, диаграммы потоков информации, схемы клиент-серверного взаимодействия, внутренней структуры мобильного приложения оператора (рис. 4). Для выбора рациональной архитектуры информационной системы и документирования построена логическая модель ПО и предметной области на языке UML. 


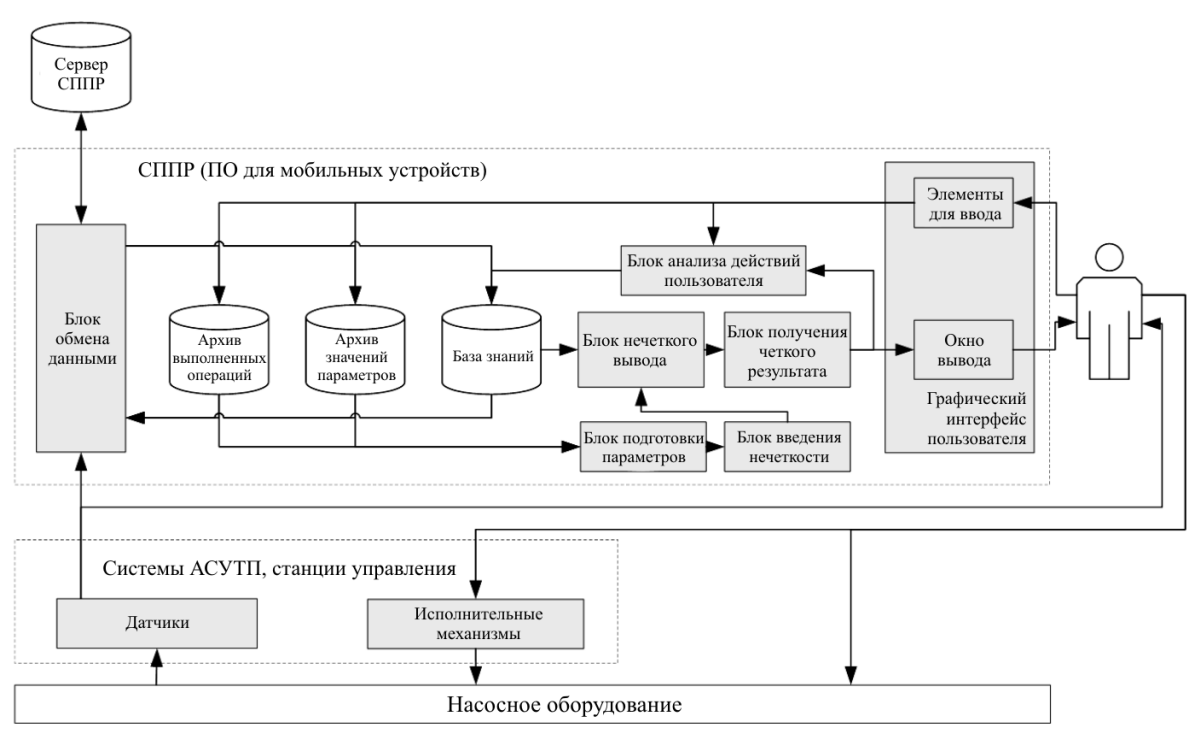

Рис. 4. Компоненты и потоки данных мобильной части информационной системы

Для численного описания лингвистических понятий и нечетких предикатов на основании экспертных оценок построены функции истинности (см. рис. 3), которые в процессе тестовой эксплуатации постоянно актуализировались.

Для реализации алгоритма работы подсистемы поддержки принятия решений использован матричный нечеткий логический вывод на основании векторных предикатов и модифицированная нечеткая комбинационная схема, как показано выше.

Полученное в результате ПО «МИРМ» было внедрено в рамках пилотного проекта на нескольких нефтяных месторождениях Пермского края. По результатам работы получены положительные отзывы от специалистов-нефтяников. Кроме того, в ходе пилотной эксплуатации системы собраны цифровые данные о работе оборудования и действиях операторов, позволяющие обучать другие виды интеллектуальных моделей.

\section{Заключение}

Привлекательность матричного подхода нечеткой логики обусловлена следующим:

1. Естественным образом обобщает логические операции на область нечетких переменных и во многих случаях адекватно моделирует нечеткие рассуждения. 
2. Позволяет производить нечеткий логический вывод с использованием правил modus ponens, modus tollens и других путем решения линейных алгебраических уравнений.

3. Позволяет проверять базу знаний на полноту с использованием точных границ области существования логического вывода.

Предложенная модель работы нечеткого логического автомата в виде модифицированной комбинационной схемы позволяет:

1. Анализировать одновременно и экспертные знания, и историю изменения значений параметров объектов.

2. Избежать использования трудноформализуемого понятия нечеткого состояния и упростить построение нечетких алгоритмов с памятью.

\section{Благодарности}

Автор выражает благодарность доктору физ.-мат. наук, профессору Михаилу Андреевичу Мариенюку за идейное вдохновение на выполнение данной работы.

Автор выражает благодарность доктору техн. наук, профессору Леониду Нахимовичу Ясницкому и канд. экон. наук Александру Олеговичу Алексееву за помощь в подготовке данной работы к публикаичии.

\section{Список литературы}

1. Камалетдинов Р.С. Механизированная добыча нефти в России: основные тенденции // Oil \& Gas Journal Russia. - 2018. - № 6. - C. 92-95.

2. Piegat A. Fuzzy modeling and control. - Heidelberg: Physica-Verlag HD: Imprint: Physica, 2001. - 728 p.

3. Djordjevic I., Petrovic D., Stojic G. A fuzzy linear programming model for aggregated production planning (APP) in the automotive industry // Computers in Industry. - 2019. - Vol. 110. - P. 48-63. DOI: 10.1016/j.compind.2019.05.004

4. Matía F., Marichal G.N., Jiménez E. Fuzzy modeling and control: theory and applications. Fuzzy modeling and control. $-2014 .-288$ p.

5. Zadeh L.A. Fuzzy sets // Information and Control. - 1965. - Vol. 8, no. 3. P. 338-353.

6. Марценюк М.А. Матричное представление нечеткой логики // Нечеткие системы и мягкие вычисления: науч. журнал Рос. ассоц. нечетких систем и мягких вычислений. - 2007. - Т. 2, № 3. - С. 7-35. 
7. Марценюк М.А., Поляков В.Б., Селетков И.П. Матричная реализация алгоритмов нечеткого вывода // Научно-технические ведомости СанктПетербургского государственного политехнического университета. Информатика. Телекоммуникации. Управление. - 2012. - Т. 6 (162). - С. 133-141.

8. Марценюк М.А., Селетков И.П. Приведение конечного нечеткого автомата к нечеткой комбинационной схеме с блоком памяти // Научнотехнические ведомости Санкт-Петербургского государственного политехнического университета. Информатика. Телекоммуникации. Управление. 2014. - T. 6 (210). - С. 67-80.

9. Круглов В.В., Дли М.И., Голунов Р.Ю. Нечеткая логика и искусственные нейронные сети. - М.: Физматлит, 2001. - 221 с.

10. González de Mendívil J.R., Figueredo F.F. Canonization of max-min fuzzy automata // Fuzzy Sets and Systems. - 2019. - Vol. 376. - P. 152-168.

11. Li Y., Wang Q. The universal fuzzy automaton // Fuzzy Sets and Systems. - 2014. - Vol. 249. - P. 27-48.

12. Stamenković A., Ćirić M., Bašić M. Ranks of fuzzy matrices. Applications in state reduction of fuzzy automata // Fuzzy Sets and Systems. - 2018. Vol. 333. - P. 124-139.

13. Брауэр В. Введение в теорию конечных автоматов. - М.: Радио и связь, 1987. -392 с.

14. Карпов Ю.Г. Теория автоматов. - СПб., 2003. - 208 с.

15. Марценюк М.А. Операторно-логические схемы как средство изучения алгоритмов в учебных курсах по математике и информатике // Прикладная информатика. - 2010. - № 5 (29). - С. 43-54.

\section{References}

1. Kamaletdinov R.S. Mekhanizirovannaia dobycha nefti v Rossii: osnovnye tendentsii [Mechanized oil production in Russia: main trends]. Oil \& Gas Journal Russia, 2018, no. 6, pp. 92-95.

2. Piegat A. Fuzzy Modeling and Control. - Heidelberg: Physica-Verlag HD: Imprint: Physica, 2001, 728 p.

3. Djordjevic I., Petrovic D., Stojic G. A fuzzy linear programming model for aggregated production planning (APP) in the automotive industry. Computers in Industry, 2019, vol. 110, pp. 48-63. DOI: 10.1016/j.compind.2019.05.004.

4. Matía F., Marichal G.N., Jiménez E. Fuzzy modeling and control: theory and applications. Fuzzy modeling and control, 2014, 288 p.

5. Zadeh L.A. Fuzzy sets. Information and Control, 1965, vol. 8, no. 3, pp. 338-353.

6. Martsenyuk M.A. Matrix Representation of Fuzzy Logic. Fuzzy Systems and Soft Computing. Science Journal of Russian Association of Fuzzy Systems and Soft Computing, 2007, vol. 2, no. 3, pp. 7-35. 
7. Martsenyuk M.A., Polyakov V.B., Seletkov I.P. Matrix Implementation of Fuzzy Inference Algorithms. St. Petersburg State Polytechnical University Journal. Computer Science. Telecommunications and Control System, 2012, no. 6 (162). pp. 133-141.

8. Martsenyuk M.A., Seletkov I.P. Conversion of Fuzzy Finite Automata to Fuzzy Combinational Circuit. St. Petersburg State Polytechnical University Journal. Computer Science. Telecommunications and Control System, 2014, no. 6 (210), pp. 67-80.

9. Kruglov V.V., Dli M.I., Golunov R.U. Nechetkaia logika i iskusstvennye neironnye seti [Fuzzy logic and artificial neural networks]. 2001, 221 p.

10. González de Mendívil J.R., Figueredo F.F. Canonization of max-min fuzzy automata. Fuzzy Sets and Systems, 2019, vol. 376, pp. 152-168.

11. Li Y., Wang Q. The universal fuzzy automaton. Fuzzy Sets and Systems, 2014, vol. 249, pp. 27-48.

12. Stamenković A., Ćirić M., Bašić M. Ranks of fuzzy matrices. Applications in state reduction of fuzzy automata. Fuzzy Sets and Systems, 2018, vol. 333, pp. 124-139.

13. Brauer W. Automatentheorie. Stuttgart: Teubner, 1984.

14. Karpov U.G. Teoriya avtomatov [Theory of the state machines]. St. Petersburg: Piter, 2003, 208 p.

15. Martsenyuk M.A. Operatorno-logicheskie shemi kak sredstvo isucheniya algoritmov v ychebnih kursah po matematike I informatike [Operator-logic diagrams as a tool for studying algorithms in training courses in mathematics and computer science]. Science journal of applied informatics, 2010, no. 5 (29), pp. 43-54.

Статья получена: 06.10.2020

Статья принята: 16.11.2020

\section{Сведения об авторе}

Селетков Илья Павлович (Пермь, Россия) - старший преподаватель кафедры «Радиоэлектроника и защита информации», инженер кафедры «Прикладная математика и информатика», Пермский государственный национальный исследовательский университет (614990, Пермь, ул. Букирева, 15, e-mail: iseletkov@gmail.com).

\section{About the author}

Ilya P. Seletkov (Perm, Russian Federation) - Senior Lecturer, Department of Radio Electronics and Information Protection, Engineer, Department of Applied Mathematics and Informatics, Perm State University (15, Bukireva st., Perm, 614990, e-mail: iseletkov@gmail.com). 
Библиографическое описание статьи согласно ГОСТ Р 7.0.100-2018:

Селетков, И.П. Применение матричного аппарата нечеткой логики для поддержки принятия решений в процессе обслуживания технологического оборудования нефтедобычи / И.П. Селетков. - DOI 10.15593/2499-9873/2020.4.05. - Текст: непосредственный // Прикладная математика и вопросы управления = Applied Mathematics and Control Sciences. - 2020. - № 4. - C. 65-88.

\section{Цитирование статьи в изданиях РИНЦ:}

Селетков И.П. Применение матричного аппарата нечеткой логики для поддержки принятия решений в процессе обслуживания технологического оборудования нефтедобычи // Прикладная математика и вопросы управления. - 2020. - № 4. C. 65-88. DOI: 10.15593/2499-9873/2020.4.05

\section{Цитирование статьи в references и международных изданиях:}

\section{Cite this article as:}

Seletkov I.P. Application of matrix approach of fuzzy logic for decision support in oil mining equipment service. Applied Mathematics and Control Sciences, 2020, no. 4, pp. 65-88. DOI: 10.15593/2499-9873/2020.4.05 (in Russian) 